\title{
Comparative study of analytical and numerical algorithms for designing reinforced concrete sections under biaxial bending
}

\author{
J.L. Bonet ${ }^{\text {a }}$, M.H.F.M. Barros ${ }^{\mathrm{b}}$, M.L. Romero ${ }^{\mathrm{c}, *}$ \\ ${ }^{a}$ Civil Engineering Department, Polytechnic University of Valencia, Spain \\ ${ }^{\mathrm{b}}$ Department of Civil Engineering, Faculty of Sciences and Technology, University of Coimbra, Portugal \\ ${ }^{\mathrm{c}}$ Department of Continuous Medium Mechanics and Theory of Structures, Polytechnic University of Valencia, Spain
}

Received 29 July 2005; accepted 7 August 2006

Available online 27 October 2006

\begin{abstract}
This paper presents a comparative study of different integration methods of stresses (both analytical and numerical) for concrete sections subjected to axial loads and biaxial bending. Such methods are applied to circular and rectangular sections. The constitutive equation used is a parabola-rectangle from the Eurocode-2. The comparison was performed with regard to the accuracy and the computational speed of each method. The objective of the paper is to determine which of the integration methods compared is more efficient in computing the interaction surfaces for rectangular and circular sections. The analytical method proposed by Barros et al. [Barros MHFM, Barros A, Ferreira C. Closed form solution of optimal design of rectangular reinforced concrete sections. Eng Comput 2004;21(7):761-76] for rectangular sections is compared with the numerical method termed "modified thick layer integration" proposed by Bonet et al. [Bonet JL, Romero ML, Miguel PF, Fernandez MA. A fast stress integration algorithm for reinforced concrete sections with axial loads and biaxial bending. Comput Struct 2004;82(2-3):213-25] and with the well-known fiber method. Furthermore, two new methods are proposed for circular sections: one analytical and one numerical based on the Gauss-Legendre quadrature. The results of both methods are compared with the classical layer decomposition method.
\end{abstract}

(C) 2006 Elsevier Ltd. All rights reserved.

Keywords: Stress integration; Reinforced concrete; Cross-section analysis; Short columns; Biaxial bending; Ultimate design

\section{Introduction}

Computer programs for designing reinforced concrete structures need to compute the internal forces through the integration of stresses over the concrete cross-section in order to obtain the interaction surface $\left(N_{u}, M_{u y}, M_{u z}\right)$. This action is performed many times, and thus its optimisation represents an important reduction in computing time. Romero et al. [3] demonstrated that the runtime to obtain the internal forces of the section is mainly consumed during the evaluation of the internal equilibrium forces of concrete sections $\left(N_{\mathrm{c}}, M_{\mathrm{c} y}, M_{\mathrm{c} z}\right)$ and not in the steel bars.

\footnotetext{
* Corresponding author. Tel.: +3496387 7007; fax: +34963879679.

E-mail address: mromero@mes.upv.es (M.L. Romero).
}

Generally, the columns of such structures are subjected to axial loads and biaxial bending moments as a result of their geometry, the shape of the cross-section or the type of external forces exerted. In edification many examples follow this behaviour such as the columns in the corners of building and elements affected by seismic and wind forces. For this type of structures the sections are typically rectangular or circular. The classical method of integrating the stresses $[4,5]$, etc.) in the concrete is usually performed by dividing the section into layers or fibers (also called cells). This technique is not numerically efficient due to the huge amount of information that is required to characterise the section and the large number of numerical operations needed to reach an acceptable level of error, Miguel et al. [6] . Moreover, this integration method could produce convergence problems for non-linear structural analysis 
programs, since the variation of the internal forces is not continuous for different locations of the neutral axis and depends on the fiber mesh size or the layer height. There are a lot of alternative methods to integrate the concrete stresses over the cross-section, some of the most interesting of which are those by Basu [7], Bröndum [8,9] and Yen [10]. These authors study the integration of stresses if the constitutive equation is the equivalent rectangular stress block. Rodríguez and Aristizabal [11] also proposed computing such integrals by decomposing the section into many trapezes. In this case the constitutive equation is a parabola-rectangle diagram for the ascending branch and linear for the descending branch. They obtain the solution to the stresses integral analytically. Recently Barros et al. [1] obtained the stress integral for the rectangular section analytically using the Heaviside functions. This method is valid for ultimate loads, axial load and uniaxial bending and a parabola-rectangle diagram. Previously, the same authors, Barros et al. [12], used the constitutive equation of Model Code 90 [14] for a non-linear structural analysis under axial load and biaxial bending.

Fafitis [13] developed a method for computing the internal forces of the concrete using Green's theorem. Use of this method allows the stress integral of the compressed area to be transformed into a path integral over the perimeter. This integral is solved numerically using the GaussLegendre quadrature. Bonet et al. [2] also proposed two methods based on the Gauss quadrature. The first method was valid for a non-cylindrical stress field and the authors presented an automatic algorithm which subdivided the non-cracked concrete area with any polygonal shape (including holes) into a small number of quadrilateral areas. The second method is suitable only for polygonal sections (including holes) in which the stress field is uniform over one direction (cylindrical stress fields) and it was decomposed the integration area into thick (or wide) layers parallel to the neutral axis. The integral of each layer was transformed into a path integral along the perimeter. This last method was termed the "Modified Thick Layer Integration" (MTLI) method. In this former paper, Bonet et al [2] compared these two new methods based on the Gauss quadrature, the fiber method and the method proposed by Fafitis [13] in terms of accuracy and speed (efficiency). In this study, the constitutive equation respected was the Model Code 90 [14] for a non linear anal- ysis. These authors concluded the method most efficient is the MTLI.

\section{Objectives and scope}

In this research a comparative study of different analytical and numerical methods to integrate the concrete stresses over cross section subjected to axial loads and biaxial bending is performed. The studied methods are: classical decomposition fibers or layers, analytical using the Heaviside functions and numerical based on Gauss-Legendre quadrature.

The objective is to find out which is the most efficient integration algorithm for the calculation of the interaction surface $\left(N_{u}, M_{u x}, M_{u y}\right)$ of a reinforced concrete section. The efficiency of the integration methods have been studied in terms of accuracy and speed, and they have been applied to both circular and rectangular sections.

In this paper the analytical method using the Heaviside functions proposed for rectangular sections by Barros et al. [1] and the numerical method for the polygonal section by Bonet et al. [1] are explained. These methods will be compared with the results obtained from fibers decomposition method. Furthermore, two new methods are introduced for the circular section: analytical using the Heaviside functions and numerical based on the Gauss-Legendre quadrature. These last methods will be compared with the layers decomposition method.

In order to obtain the ultimate capacity of the concrete section $\left(N_{\mathrm{c}}, M_{\mathrm{c} y}, M_{\mathrm{c} z}\right)$, the stresses associated to each ultimate plane of strain (defined by the pivot diagram) are integrated (Art 6.1. Euro Code-2 [December 2004] [15]). The concrete constitutive equation used in this study is a parabola-rectangle for normal strength concrete, Fig. 1.

\section{Analytical integration methods}

\subsection{Ultimate section deformation}

The strains in a reinforced concrete section vary linearly, being equal to zero at the neutral axis, defined by its position ' $X$ ", or the non-dimensional variable $\alpha$ given by

$\alpha=\frac{X}{d}$

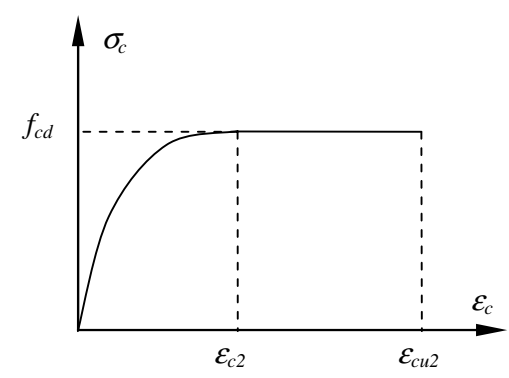

$$
\begin{gathered}
\text { a) For } 0 \leq \varepsilon_{c} \leq \varepsilon_{c 2} \quad \sigma_{c}=f_{c d} \cdot\left[1-\left(1-\frac{\varepsilon_{c}}{\varepsilon_{c 2}}\right)^{2}\right] \\
\text { b) For } \varepsilon_{c 2} \leq \varepsilon_{c} \leq \varepsilon_{c u 2} \quad \sigma_{c}=f_{c d} \\
\text { where : } \varepsilon_{c 2}(\% o)=2.0 ; \quad \varepsilon_{c u 2}(\% o)=3.5
\end{gathered}
$$

Fig. 1. Parabola-rectangle diagram. 

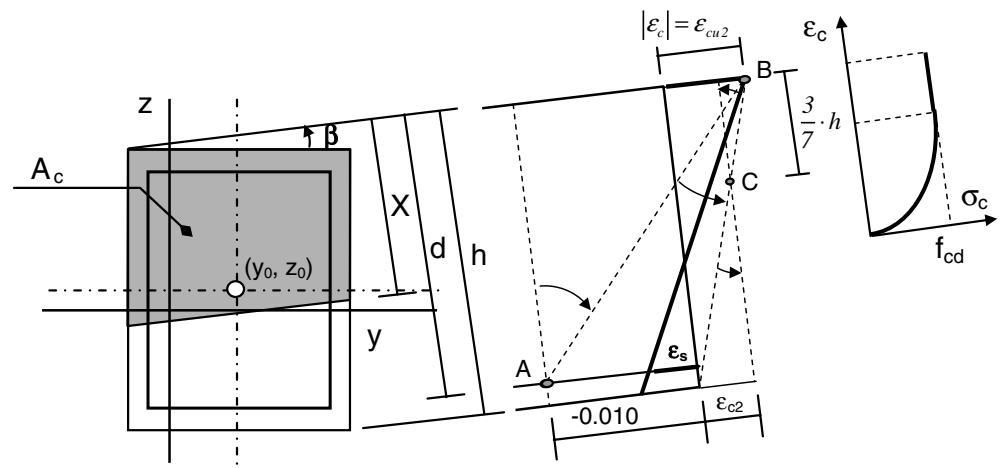

a

b

Fig. 2. Failure of the rectangular section: (a) Pivot diagram. Ultimate deformation of the section when the neutral axis has a depth "X" and (b) stress distribution.

where " $d$ " is the effective height The initial and deformed plane sections are represented in Fig. 2.

The failure of the section can occur in the following ways (Fig. 2): (a) due to the concrete, when the maximum strain $\varepsilon_{\mathrm{c}}$ is equal to the ultimate strain value $\varepsilon_{\mathrm{cu} 2}$; (b) due to the steel, when the strain in steel $\varepsilon_{\mathrm{s}}$ is equal to the maximum strain $1 \%$; and (c) with a limit compression of $0.2 \%$ when the section is all under compression. In the case of a circular section, the deformations at rupture are represented in Fig. 3. These situations of rupture are described using Heaviside functions, as in Barros et al. [1], by the following continuous equations:

$$
\begin{aligned}
\varepsilon_{\mathrm{c}}= & \frac{1}{100} \frac{\alpha}{\alpha-1}-H\left(\alpha-\frac{\varepsilon_{\mathrm{cu} 2}}{\varepsilon_{\mathrm{cu} 2}+1 \%}\right)\left(\varepsilon_{\mathrm{cu} 2}+\frac{\alpha}{100(\alpha-1)}\right) \\
& +H(X-h)\left(\varepsilon_{\mathrm{cu} 2}-\frac{X}{X-3 / 7 h} 0.2 \%\right) \\
\varepsilon_{\mathrm{s}}= & \varepsilon_{\mathrm{c}} \frac{d-X}{X}
\end{aligned}
$$

where the Heaviside function $H(x)$ can be defined by either of:

$H(x)= \begin{cases}1 & x>0 \\ 0 & x<0\end{cases}$

\subsection{Equilibrium equations}

The resulting force in the compressed concrete $N_{\mathrm{c}}$ is obtained by the integration of the stresses $\sigma_{\mathrm{c}}(y, z)$ in the compressed area $A_{\mathrm{c}}$, represented by the dashed area in Fig. 4. The corresponding bending moments $M_{\mathrm{c} y}$ and $M_{\mathrm{c} z}$ are obtained by the same integration procedure, Eq. (4).

$$
\begin{aligned}
& N_{\mathrm{c}}=\iint_{A_{\mathrm{c}}} \sigma_{\mathrm{c}}(y, z) \mathrm{d} y \mathrm{~d} z \\
& M_{\mathrm{c} y}=\iint_{A_{\mathrm{c}}} \sigma_{c}(y, z)\left(z-z_{0}\right) \mathrm{d} y \mathrm{~d} z \\
& M_{\mathrm{c} z}=\iint_{A_{\mathrm{c}}} \sigma_{c}(y, z)\left(y-y_{0}\right) \mathrm{d} y \mathrm{~d} z
\end{aligned}
$$

where $\left(y_{0}, z_{0}\right)$ are the coordinates of the centre of reference for the internal forces of the section.

\subsection{Rectangular section}

The integration is performed over the different compression areas indicated in Fig. 4. These results of the integration depend on the variables $X$ and $\beta$, Fig. $4 \mathrm{a}$ and the Heaviside functions. In the work developed by Barros et al. [12] the complete expressions for the case of the
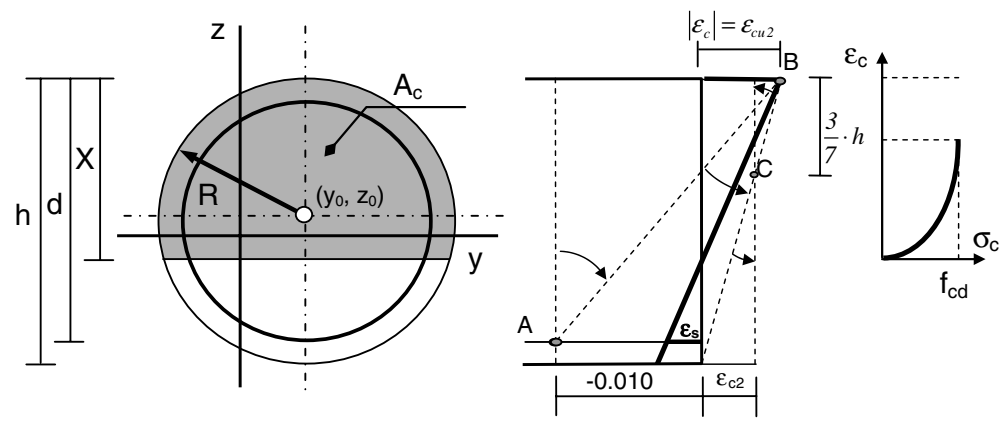

a

b

Fig. 3. Failure of the circular section: (a) Pivot diagram. Ultimate deformation of the section when the neutral axis has a depth "X" and (b) stress distribution. 


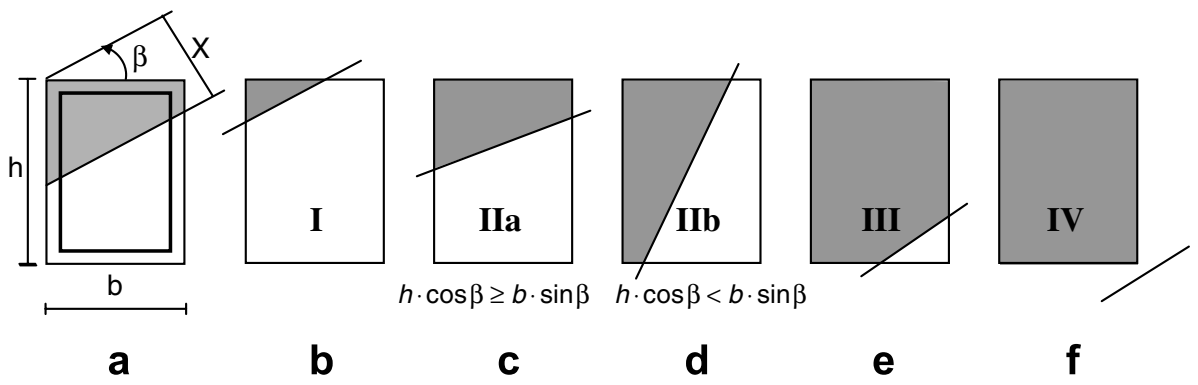

Fig. 4. Concrete compression area Ac.

non-linear equation of the CEB-FIP Model Code 1990 [14] are indicated.

\subsubsection{Separated components}

The integration of the previous equations in each of the domains indicated from Fig. $4 \mathrm{~b}-\mathrm{f}$ results in the following axial loads: $N_{\text {cI }}, N_{\text {cII }}, N_{\text {cIIb }}, N_{\text {cIII }}$ and $N_{\text {cIV }}$. The same procedure is used with the bending moment $M_{\mathrm{c} y}$ to give $M_{\mathrm{c} y \mathrm{I}}$, $M_{\mathrm{c} y I I \mathrm{a}}, M_{\mathrm{c} y I \mathrm{Ib}}, M_{\mathrm{c} y I I I}$ and $M_{\mathrm{c} y I \mathrm{~V}}$. The integration regarding $M_{\mathrm{c} z}$ gives the result $M_{\mathrm{c} z \mathrm{I}}, M_{\mathrm{c} z I I \mathrm{a}}, M_{\mathrm{c} z \mathrm{II} b}, M_{\mathrm{c} z I I I}$ and $M_{\mathrm{c} z \mathrm{IV}}$.

\subsubsection{Unique equations}

The use of Heaviside functions allows a unique expression to be written considering the five integration areas from Fig. 4. This expression is as follows in the case of the axial load:

$N_{\mathrm{c}}=$ Heaviside $(h \cos \beta-X) \cdot \operatorname{Heaviside}(b \sin \beta-X)$

$\cdot$ Heaviside $(X) \cdot N_{\mathrm{cI}}+\operatorname{Heaviside}(b \sin \beta-X)$

$\cdot$ Heaviside $(X-h \cos \beta) \cdot \operatorname{Heaviside}(X) \cdot N_{\text {cIIa }}$

+ Heaviside $(h \cos \beta-X) \cdot \operatorname{Heaviside}(X-b \sin \beta)$

- Heaviside $(X) \cdot N_{\text {cIIb }}+$ Heaviside $(b \sin \beta+h \cos \beta-X)$

- Heaviside $(X-b \sin \beta) \cdot \operatorname{Heaviside}(X)$

- Heaviside $(X-h \cos \beta) \cdot N_{\text {cIII }}$

+ Heaviside $(X-b \sin \beta-h \cos \beta)$

$\cdot \operatorname{Heaviside}(X) \cdot N_{\mathrm{cIV}}$

Equivalent equations can be written for the bending moments $M_{\mathrm{c} y}$ and $M_{\mathrm{c} z}$ giving the following results, respectively:

$$
\begin{aligned}
M_{\mathrm{cy}}= & \text { Heaviside }(h \cos \beta-X) \cdot \operatorname{Heaviside}(b \sin \beta-X) \\
& \cdot \text { Heaviside }(X) \cdot M_{\mathrm{cyI}}+\operatorname{Heaviside}(b \sin \beta-X) \\
& \cdot \text { Heaviside }(X-h \cos \beta) \cdot \operatorname{Heaviside}(X) \cdot M_{\mathrm{cyIII}} \\
& +\operatorname{Heaviside}(h \cos \beta-X) \cdot \operatorname{Heaviside}(X-b \sin \beta) \\
& \cdot \text { Heaviside }(X) \cdot M_{\mathrm{cyIIb}}+\operatorname{Heaviside}(b \sin \beta+h \cos \beta-X) \\
& \cdot \text { Heaviside }(X-b \sin \beta) \cdot \operatorname{Heaviside}(X) \\
& \cdot \text { Heaviside }(X-h \cos \beta) \cdot M_{\mathrm{cyIII}} \\
& + \text { Heaviside }(X-b \sin \beta-h \cos \beta) \cdot \operatorname{Heaviside}(X) \cdot M_{\mathrm{cyIV}}
\end{aligned}
$$

$M_{\mathrm{c} z}=$ Heaviside $(h \cos \beta-X) \cdot$ Heaviside $(b \sin \beta-X)$

$$
\begin{aligned}
& \cdot \text { Heaviside }(X) \cdot M_{\text {czI }}+\text { Heaviside }(b \sin \beta-X) \\
& \cdot \text { Heaviside }(X-h \cos \beta) \cdot \operatorname{Heaviside}(X) \cdot M_{\text {czIIa }} \\
& + \text { Heaviside }(h \cos \beta-X) \cdot \operatorname{Heaviside}(X-b \sin \beta) \\
& \cdot \text { Heaviside }(X) \cdot M_{\text {czIl }}+\operatorname{Heaviside}(b \sin \beta+h \cos \beta-X) \\
& \cdot \text { Heaviside }(X-b \sin \beta) \cdot \operatorname{Heaviside}(X) \\
& \cdot \text { Heaviside }(X-h \cos \beta) \cdot M_{\text {czIII }} \\
& + \text { Heaviside }(X-b \sin \beta-h \cos \beta) \cdot \operatorname{Heaviside}(X) \cdot M_{\text {czIV }}
\end{aligned}
$$

The use of these unique equations results in heavy computation times.

\subsection{Circular section}

The resulting force $N_{\mathrm{c}}$ in the compressed concrete for the circular section represented in Fig. 3 and the bending moments $M_{\mathrm{c} y}$ and $M_{\mathrm{c} z}$ are computed using Eq. (5). The concrete compression zone $A_{\mathrm{c}}$ is represented in Fig. 5a, where the neutral axis intersects the section (denoted by case I with resulting force $N_{\mathrm{cI}}$ and the bending moments $M_{\text {cyI }}$ and $M_{\text {zI }}$ ) and in Fig. $3 \mathrm{~b}$, where it is located outside the section (case II with resulting force and the bending moments $M_{\text {cyII }}$ and $M_{\text {czII }}$ ).

The results obtained are introduced into unique equations by the use of Heaviside functions giving the total force and the bending moments, respectively: a

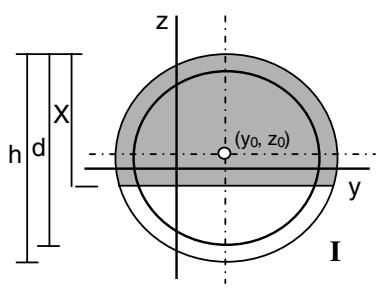

b

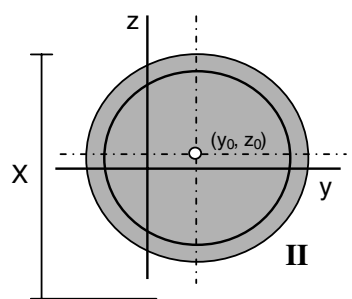

Fig. 5. Concrete compression zone Ac in a circular section: (a) neutral axis crossing the section and (b) neutral axis outside the section. 
a

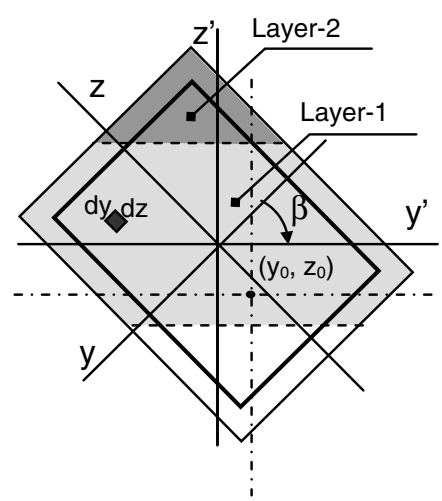

b

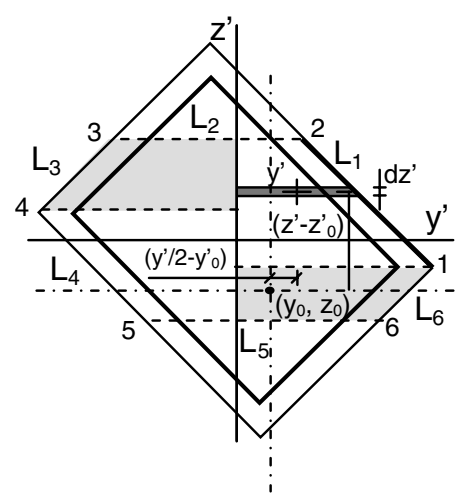

C

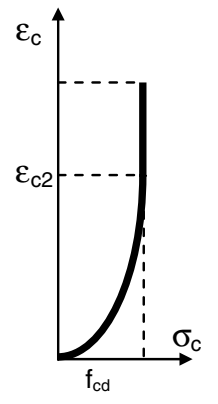

Fig. 6. Numerical integration method. Polygonal section: (a) decomposition into thick layers, (b) Decomposition into "nL" quadrilaterals and transformation into a path integral and (c) concrete stress distribution.

$$
\begin{aligned}
N_{\mathrm{c}}= & N_{\mathrm{cI}} \cdot \operatorname{Heaviside}(2 R-X) \cdot \operatorname{Heaviside}(X) \\
& +N_{\mathrm{cII}} \cdot \operatorname{Heaviside}(X-2 R) \\
M_{\mathrm{c} y}= & M_{\mathrm{cyI}} \cdot \operatorname{Heaviside}(2 R-X) \cdot \operatorname{Heaviside}(X) \\
& +M_{\mathrm{cyII}} \cdot \operatorname{Heaviside}(X-2 R) \\
M_{\mathrm{c} z}= & M_{\mathrm{czI}} \cdot \operatorname{Heaviside}(2 R-X) \cdot \operatorname{Heaviside}(X) \\
& +M_{\mathrm{cz} I \mathrm{I}} \cdot \operatorname{Heaviside}(X-2 R)
\end{aligned}
$$

where $R$ is the radius of the section.

\section{Numerical integration methods}

\subsection{Polygonal section}

The internal forces of the concrete section are obtained from Eq. (5). The integral of stresses over the non-cracked area is determined numerically by applying the "modified thick layer integration" (MTLI) method, Bonet et al. [1].

The method is explained below applied to a parabolarectangle diagram, Fig. 1.

\subsubsection{Decomposition into thick layers}

When the concrete stress function is not defined by a unique and polygonal equation, it is useful to divide the compressed concrete area $A_{\mathrm{c}}$ into thick layers (also called wide layers) parallel to the neutral axis with the purpose of achieving better accuracy in the resolution of the numerical integration. Bearing in mind the constitutive equation used in this paper (Fig. 6) is not defined by unique equation, if there are concrete fibers in the compressed area with a strain higher than the ultimate deformation under simple compression $\left(\varepsilon_{\mathrm{c} 2}\right)$ and also fibers with strains lower than $\varepsilon_{\mathrm{c} 2}$, the area is divided in two layers. The first layer corresponds to the group of fibers with strains below $\varepsilon_{\mathrm{c} 2}$, and the second layer corresponds to the fibers with a deformation above $\varepsilon_{\mathrm{c} 2}$ (Fig. 6a). Otherwise, it is not necessary to decompose the section into thick layers.

The internal forces of the concrete $\left(N_{\mathrm{c}}, M_{\mathrm{c} y}, M_{\mathrm{c} z}\right)$ are obtained by addition of the internal forces of each thick layer $\left(N_{\mathrm{c} i}, M_{\mathrm{c} y i}, M_{\mathrm{c} z i}\right)$ into which the integration area $A_{\mathrm{c}}$ is decomposed:

$N_{\mathrm{c}}=\sum_{i=1}^{\mathrm{nt}} N_{\mathrm{c} i} ; \quad M_{\mathrm{c} y}=\sum_{i=1}^{\mathrm{ntl}} M_{\mathrm{c} y i} ; \quad M_{\mathrm{c} z}=\sum_{i=1}^{\mathrm{ntl}} M_{\mathrm{c} z i}$

where $n t l$ is the number of thick layers (one or two depending of the fiber with strain higher).

\subsubsection{Transformation into a path integral}

For each of the thick layers, the stress integral can be transformed into a path integral along the perimeter of the integration zone (Fig. 6b) because the concrete stress field has a preferred direction (orthogonal to the neutral axis).

$$
\begin{aligned}
& N_{\mathrm{c}}=\sum_{i=1}^{\mathrm{ntl}}\left[\oint_{L} y^{\prime} \sigma\left(z^{\prime}\right) \mathrm{d} z^{\prime}\right]_{i} \\
& M_{\mathrm{c} y}=M_{\mathrm{c} y}^{\prime} \cdot \cos \beta-M_{\mathrm{c} z}^{\prime} \cdot \sin \beta \\
& M_{\mathrm{c} z}=M_{\mathrm{c} y}^{\prime} \cdot \sin \beta+M_{\mathrm{c} z}^{\prime} \cdot \cos \beta
\end{aligned}
$$

where $\beta$ is the inclination angle of the neutral axis (Fig. 2) and

$$
\begin{aligned}
& M_{\mathrm{c} y}^{\prime}=\sum_{i=1}^{\mathrm{ntl}}\left[\oint_{L} \sigma_{\mathrm{c}}\left(z^{\prime}\right)\left(z^{\prime}-z_{0}^{\prime}\right) \mathrm{d} z^{\prime}\right]_{i} \\
& M_{\mathrm{c} z}^{\prime}=\sum_{i=1}^{\mathrm{ntl}}\left[\oint_{L} \sigma_{\mathrm{c}}\left(z^{\prime}\right)\left(y^{\prime} / 2-y_{0}^{\prime}\right) \mathrm{d} z^{\prime}\right]_{i}
\end{aligned}
$$

$\left(y_{0}^{\prime}, z_{0}^{\prime}\right)$ are the coordinates of the centre of reference for the internal forces of the section for the axes $\left(y^{\prime}, z^{\prime}\right)$.

\subsubsection{Evaluation of the stress integral by applying the Gauss-Legendre quadrature}

Due to the fact that the perimeter of each layer is polygonal, the integral along the contour " $L$ " can be computed as a sum of the integrals of each side of the perimeter (Fig. 7): 


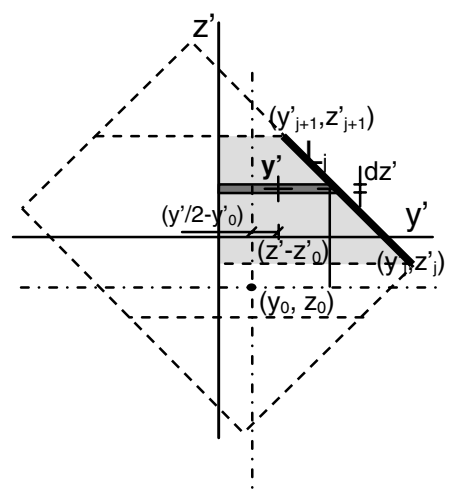

Fig. 7. Evaluation of the concrete stresses integral.

$\oint_{L} h\left(z^{\prime}\right) \mathrm{d} z^{\prime}=\sum_{j=1}^{\mathrm{nL}} S_{L j}\left(z^{\prime}\right)$

where $\mathrm{nL}$ is the number of sides for the perimeter of each layer.

$S_{L j}\left(z^{\prime}\right)=\int_{z_{j}}^{z_{j+1}} h\left(z^{\prime}\right) \mathrm{d} z^{\prime}$

The "side by side" integral can be performed by the Gauss-Legendre quadrature integration method:

$S_{L j}\left(z^{\prime}\right)=\frac{\left(z_{j+1}^{\prime}-z_{j}^{\prime}\right)}{2} \cdot \sum_{k=1}^{\text {ngp }} \omega_{k} \cdot h\left(\xi_{k}\right)$

where $\xi_{k}$ is the value of the curvilinear coordinate of the Gauss point ' $k$ '; $\omega_{k}$ is the weight associated to such a Gauss point; and "ngp" is the number of Gauss points considered in the integral.

This algorithm allows the stresses of a hollow or a solid section to be integrated. To do so, we only have to enumerate the vertices of the polygon in an anticlockwise (positive) or clockwise (negative) direction.

\subsection{Circular section}

The internal forces of the stresses of the circular section are computed from the following expressions, Fig. 8:
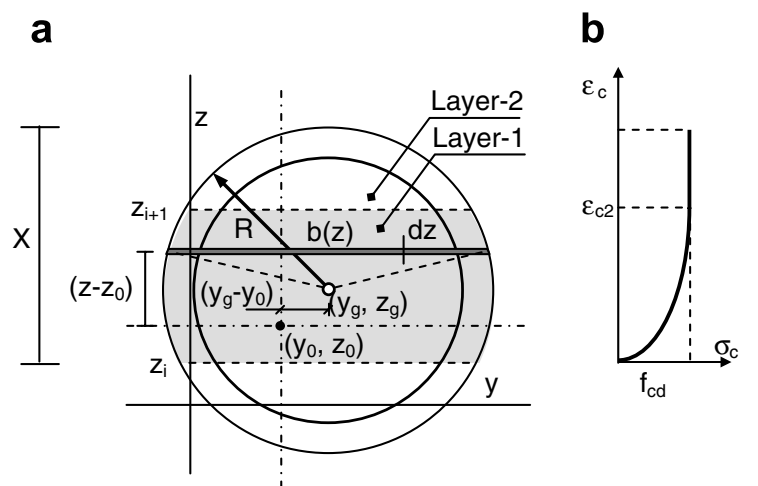

Fig. 8. Numerical integration method. Circular Section: (a) decomposition into thick layers and transformation into a path integral and (b) concrete stress distribution.

$$
\begin{aligned}
N_{\mathrm{c}} & =\iint_{A_{\mathrm{c}}} \sigma_{\mathrm{c}}(z) \mathrm{d} A=\int_{z_{g}+R-X}^{z_{g}+R} b(z) \cdot \sigma_{\mathrm{c}}(z) \mathrm{d} z=\int_{z_{g}+R-X}^{z_{g}+R} h_{1}(z) \mathrm{d} z \\
M_{\mathrm{c} y} & =\iint_{A_{\mathrm{c}}} \sigma_{\mathrm{c}}(z) \cdot\left(z-z_{0}\right) \mathrm{d} A=\int_{z_{g}+R-X}^{z_{g}+R} b(z) \cdot\left(z-z_{0}\right) \cdot \sigma_{\mathrm{c}}(z) \mathrm{d} z \\
& =\int_{z_{g}+R-X}^{z_{g}+R} h_{2}(z) \mathrm{d} z \\
M_{\mathrm{c} z} & =\iint_{A_{\mathrm{c}}} \sigma_{\mathrm{c}}(z) \cdot\left(y_{g}-y_{0}\right) \mathrm{d} A=\int_{z_{g}+R-X}^{z_{g}+R} b(z) \cdot\left(y_{g}-y_{0}\right) \cdot \sigma_{\mathrm{c}}(z) \mathrm{d} z \\
& =\int_{z_{g}+R-X}^{z_{g}+R} h_{3}(z) \mathrm{d} z
\end{aligned}
$$

where $A_{\mathrm{c}}$ is the compressed area of the concrete; $\left(y_{g}, z_{g}\right)$ are the coordinates of the centroid of the circular section; $\sigma_{\mathrm{c}}(z)$ is the concrete stress in terms of the coordinate " $z$ "; $b(z)$ is the width of the section in terms of ' $z$ ', being:

$b(z)=2 \cdot \sqrt{\left(R-z+z_{g}\right) \cdot\left(R+z-z_{g}\right)}$

In Eq. (14), the $y$-axis was chosen parallel to the neutral axis. As the concrete stress field has a preferred direction (orthogonal to the neutral axis), the stress integral of the compressed area of the concrete $A_{\mathrm{c}}$ can be transformed into a path integral in terms of ' $z$ ', from the most compressed border of the section to the position of the neutral axis.

The internal forces of the concrete, Eq. (14), are computed using the Gauss-Legendre quadrature. To do so, a similar methodology to the one used for the polygonal section will be followed.

\subsubsection{Decomposition into thick layers}

The accuracy of the integrals depends on the number of Gauss points used, on the concrete constitutive equation and on the shape of the cross-section (circular). Since the product $\left[\sigma_{\mathrm{c}}(z) \cdot b(z)\right]$ is no longer a unique and polygonal equation, it is necessary to use a large number of Gauss points for the evaluation of integrals in order to obtain a suitable degree of accuracy.

In this case, it is worth subdividing the integration area $A_{\mathrm{c}}$ into thick layers parallel to the neutral axis (Fig. 8a). The technique to define the thick layers is the same as for the polygonal section.

\subsubsection{Evaluation of the stresses integral}

To perform each of the integrals, the Gauss method is used. The following coordinate transformation is carried out, Fig. 8a:

$z=\frac{\left(z_{i+1}-z_{i}\right)}{2} \cdot(\xi+1)+z_{i} \Rightarrow \mathrm{d} z=\frac{\left(z_{i+1}-z_{i}\right)}{2} \mathrm{~d} \xi$

Thus, the integrals of the internal forces are evaluated with the following expressions: 
$N_{\mathrm{c}}=\sum_{i=1}^{\mathrm{ntl}}\left[\int_{z_{i}}^{z_{i+1}} h_{1}(z) \mathrm{d} z\right]_{i}=\sum_{i=1}^{\mathrm{ntl}}\left[\frac{\left(z_{i+1}-z_{i}\right)}{2} \cdot \sum_{k=1}^{\mathrm{ngp}} \omega_{k} \cdot h_{1}\left(\xi_{k}\right)\right]_{i}$

$M_{\mathrm{cy}}=\sum_{i=1}^{\mathrm{ntl}}\left[\int_{z_{i}}^{z_{i+1}} h_{2}(z) \mathrm{d} z\right]_{i}=\sum_{i=1}^{\mathrm{ntl}}\left[\frac{\left(z_{i+1}-z_{i}\right)}{2} \cdot \sum_{k=1}^{\mathrm{ngp}} \omega_{k} \cdot h_{2}\left(\xi_{k}\right)\right]_{i}$

$M_{\mathrm{cz}}=\sum_{i=1}^{\mathrm{nt} 1}\left[\int_{z_{i}}^{z_{i+1}} h_{3}(z) \mathrm{d} z\right]_{i}=\sum_{i=1}^{\mathrm{ntl}}\left[\frac{\left(z_{i+1}-z_{i}\right)}{2} \cdot \sum_{k=1}^{\mathrm{ngp}} \omega_{k} \cdot h_{3}\left(\xi_{k}\right)\right]_{i}$

where $\xi_{k}$ is the value of the curvilinear coordinate of the Gauss point ' $k$ '; "ntl" is the number of thick layers; $\omega_{k}$ is the weight associated to such a Gauss point and "ngp" is the number of Gauss points considered in the integral.

\section{Comparative study}

The methods described in the previous sections were applied to both rectangular and circular sections. The same cases were also solved by using the well-known fiber method. For the rectangular section fibers have been used and for the circular section layers parallel to the neutral axis with fixed height have been used.

More specifically, the rectangular section analysed measured $0.50 \times 0.50 \mathrm{~m}$ and the circular section had a diameter of $0.50 \mathrm{~m}$. For both cases the mechanical cover of the reinforced bars was $0.05 \mathrm{~m}$ and the strength of the concrete was $25 \mathrm{MPa}$.

As was stated in Section 2, the constitutive equation in this study was a parabola-rectangle (Fig. 1).

To perform the integration by the Gauss quadrature, different numbers of Gauss points $(2,3,4,6,8,10,12$, $15,20,32$ and 48) were used for each of the sides of the perimeter of each thick layer that constituted the rectangular section. The same number of Gauss points was used to

Table 1

Cases analysed in the comparative study

\begin{tabular}{lll}
\hline Section type & $\begin{array}{l}\text { Inclination of the } \\
\text { neutral axis }\left(\beta^{\circ}\right)\end{array}$ & $\begin{array}{l}\text { Normalised depth of the neutral } \\
\text { axis }(\alpha=x / d)\end{array}$ \\
\hline Rectangular & $0^{\circ}, 15^{\circ}, 30^{\circ}, 45^{\circ}$ & $\begin{array}{l}0.01 ; \text { from } 0.1 \text { to } 2 \text { each } 0.1 ; 100 \\
\text { Circular }\end{array}$ \\
$0^{\circ}$ & $0.01 ;$ from 0.1 to 2 each $0.1 ; 100$ \\
\hline
\end{tabular}

Position of the neutral axis. integrate each of the layers that composed the compressed area of the circular section.

For the fiber method, the following meshes were used: $10 \times 10 ; \quad 20 \times 20 ; \quad 40 \times 40 ; \quad 100 \times 100 ; \quad 200 \times 200 \quad$ and $400 \times 400$. The number of layers taken into account in the circular section was: $10,20,40,100,200,400$ and 800 .

Different inclination angles $(\beta)$ of the neutral axis (Fig. 2) and normalised depth of the neutral axis $(\alpha=$ $X / d$ ) were considered; where " $X$ " is the depth of the neutral axis measured in the orthogonal direction to the neutral axis from the most compressed border of the cross-section; and " $d$ " is the effective depth of the reinforcement also measured in the orthogonal direction to the neutral axis from the most compressed fiber of concrete to the most tensile stressed bar (Fig. 2). The different cases for this study are summarised in Table 1.

\section{Results}

The most important results for both types of sections are analysed in this section. For each position of the neutral axis and for each integration method, the error and computing time were calculated.

It was noticed that the computing times are extremely small for both the analytical and numerical cases, and were in fact lower than what could be measured accurately by the computer. For this reason, the times presented here are the average value of five series of executions. Each of the series consisted in repeating the stress integral 1000 times.

The errors obtained for each method of computing the internal forces of the concrete $\left(N_{\mathrm{c}}, M_{\mathrm{c} y}, M_{\mathrm{c} z}\right)$ are presented in relative form with respect to the analytical solution, that is:

$\operatorname{Error}(\%)=\left|\frac{F_{i}-F_{\text {exac }}}{F_{\text {exac }}}\right| \cdot 100$

where $F_{i}$ is the value obtained for the $i$ th-case for an internal force $F$ (axial load or bending moment) and $F_{\text {exac }}$ is the exact value of the internal force $F$ (axial load or bending moment) obtained for the analytical solution.

Table 2 shows the computing time consumed for the rectangular section, as well as the maximum errors for any of the internal forces (axial load or bending moment)

Table 2

Rectangular section: Results obtained with the numerical integration

\begin{tabular}{|c|c|c|c|c|c|c|c|c|c|c|c|c|}
\hline \multirow[t]{3}{*}{$\alpha$} & \multicolumn{12}{|c|}{ Number of Gauss points for each layer } \\
\hline & \multicolumn{2}{|l|}{2} & \multicolumn{2}{|l|}{3} & \multicolumn{2}{|l|}{4} & \multicolumn{2}{|l|}{6} & \multicolumn{2}{|l|}{10} & \multicolumn{2}{|l|}{15} \\
\hline & $t(\mathrm{sg})$ & $E(\%)$ & $t(\mathrm{sg})$ & $E(\%)$ & $t(\mathrm{sg})$ & $E(\%)$ & $t(\mathrm{sg})$ & $E(\%)$ & $t(\mathrm{sg})$ & $E(\%)$ & $t(\mathrm{sg})$ & $E(\%)$ \\
\hline 0.10 & 1.141 & 0.587 & 1.282 & 0.000 & 1.503 & 0.000 & 1.822 & 0.000 & 2.574 & 0.000 & 3.405 & 0.000 \\
\hline 0.40 & 1.623 & 0.685 & 1.862 & 0.000 & 2.203 & 0.000 & 2.824 & 0.000 & 4.025 & 0.000 & 5.538 & 0.000 \\
\hline 0.80 & 1.782 & 0.102 & 2.103 & 0.000 & 2.444 & 0.000 & 3.175 & 0.000 & 4.577 & 0.000 & 6.370 & 0.000 \\
\hline
\end{tabular}


Table 3

Circular section: Results obtained with the numerical integration

\begin{tabular}{|c|c|c|c|c|c|c|c|c|c|c|c|c|}
\hline \multirow[t]{3}{*}{$\alpha$} & \multicolumn{12}{|c|}{ Number of Gauss points for each layer } \\
\hline & \multicolumn{2}{|l|}{2} & \multicolumn{2}{|l|}{3} & \multicolumn{2}{|l|}{4} & \multicolumn{2}{|l|}{6} & \multicolumn{2}{|l|}{10} & \multicolumn{2}{|l|}{15} \\
\hline & $t(\mathrm{sg})$ & $E(\%)$ & $t(\mathrm{sg})$ & $E(\%)$ & $t(\mathrm{sg})$ & $E(\%)$ & $t(\mathrm{sg})$ & $E(\%)$ & $t(\mathrm{sg})$ & $E(\%)$ & $t(\mathrm{sg})$ & $E(\%)$ \\
\hline 0.01 & 0.409 & 3.160 & 0.453 & 1.011 & 0.487 & 0.453 & 0.583 & 0.145 & 0.753 & 0.034 & 0.997 & 0.010 \\
\hline 0.10 & 0.403 & 2.828 & 0.453 & 0.950 & 0.489 & 0.429 & 0.583 & 0.138 & 0.751 & 0.032 & 0.981 & 0.010 \\
\hline 0.40 & 0.515 & 0.689 & 0.593 & 0.253 & 0.685 & 0.116 & 0.855 & 0.038 & 1.198 & 0.009 & 1.618 & 0.003 \\
\hline 0.80 & 0.505 & 2.348 & 0.599 & 0.611 & 0.683 & 0.273 & 0.849 & 0.088 & 1.196 & 0.020 & 1.624 & 0.006 \\
\hline 1.40 & 0.513 & 10.51 & 0.597 & 1.371 & 0.685 & 0.479 & 0.851 & 0.131 & 1.202 & 0.028 & 1.622 & 0.008 \\
\hline 2.00 & 0.507 & 3.445 & 0.597 & 2.907 & 0.683 & 1.382 & 0.861 & 0.450 & 1.192 & 0.105 & 1.622 & 0.033 \\
\hline
\end{tabular}

and any inclination of the neutral axis $(\beta)$, in terms of the number of Gauss points and of the relative depth of the neutral axis $(\alpha)$. In general, the maximum errors are reached for the maximum inclination of the neutral axis. Nevertheless, it can be observed that with only three Gauss points, the error is almost zero and the computing time is very small.

Table 3 presents the results of the circular section for different number of Gauss points and relative depths of the neutral axis $(\alpha)$. In comparison with the results for the rectangular section (Table 2), it was observed that for the same number of Gauss points for each layer and for the same $\alpha$, the error for the circular section is higher. This behaviour is due to the shape of the section. Furthermore, if 15 Gauss points are applied to each layer, an admissible degree of accuracy (lower than $0.1 \%$ ) is obtained with a reasonable computing time in comparison to other integration methods (Tables 5 and 7).

Tables 4 and 5 show the results from the fiber and layer integration methods respectively. With respect to the fiber method, for the rectangular section it can be inferred that for a higher relative depth of the neutral axis $\alpha$ the error decreases because the mesh of fibers adapts better to the concrete stress field. It can also be seen that for very small values of $\alpha$ the error is not admissible, because the size of the fiber is quite a lot higher than the depth of the neutral axis. In other words, the error obtained is mesh-dependent. Moreover, in comparison with the results obtained in Table 2, the execution time is quite a lot higher for the same error order.

On the other hand, with respect to the layer integration method for the circular section it can be observed that the errors increase as the relative depth of the neutral axis $(\alpha)$ decreases. In addition, for very small values of $\alpha$ the errors are not admissible. It can be seen that the error is also mesh-dependent. Finally, on comparing with the results from Table 3, the computing times are much higher for a similar error.

The times from the analytical integral for the rectangular section are shown in Table 6. If the integral is

Table 4

Rectangular section: Results obtained with the fiber method

\begin{tabular}{|c|c|c|c|c|c|c|c|c|c|c|c|c|}
\hline \multirow[t]{3}{*}{$\alpha$} & \multicolumn{12}{|c|}{ Mesh of fibers } \\
\hline & \multicolumn{2}{|c|}{$10 \times 10$} & \multicolumn{2}{|c|}{$20 \times 20$} & \multicolumn{2}{|c|}{$40 \times 40$} & \multicolumn{2}{|c|}{$100 \times 100$} & \multicolumn{2}{|c|}{$200 \times 200$} & \multicolumn{2}{|c|}{$400 \times 400$} \\
\hline & $t(\mathrm{sg})$ & $E(\%)$ & $t(\mathrm{sg})$ & $E(\%)$ & $t(\mathrm{sg})$ & $E(\%)$ & $t(\mathrm{sg})$ & $E(\%)$ & $t(\mathrm{sg})$ & $E(\%)$ & $t(\mathrm{sg})$ & $E(\%)$ \\
\hline 0.01 & 26.04 & 100 & 71.7 & 100 & 274.4 & 100 & 1723 & 19.33 & 6779 & 18.02 & 27179 & 7.108 \\
\hline 0.10 & 25.53 & 19.52 & 72.8 & 16.28 & 276.9 & 16.50 & 1753 & 0.623 & 6780 & 0.631 & 27189 & 0.633 \\
\hline 0.40 & 26.24 & 36.40 & 75.01 & 9.610 & 286.8 & 9.441 & 1783 & 0.089 & 7040 & 0.131 & 28220 & 0.133 \\
\hline 0.80 & 27.94 & 10.52 & 79.22 & 9.846 & 306.7 & 2.229 & 1853 & 0.037 & 7531 & 0.111 & 30284 & 0.108 \\
\hline 1.40 & 28.55 & 1.675 & 81.51 & 0.421 & 313.2 & 0.105 & 1952 & 0.189 & 7751 & 0.004 & 30914 & 0.001 \\
\hline 2.00 & 28.34 & 1.675 & 81.52 & 0.421 & 313.2 & 0.105 & 1963 & 0.017 & 7801 & 0.004 & 30854 & 0.003 \\
\hline
\end{tabular}

Table 5

Circular Section: Results obtained with the layer method

\begin{tabular}{|c|c|c|c|c|c|c|c|c|c|c|c|c|}
\hline \multirow[t]{3}{*}{$\alpha$} & \multicolumn{12}{|c|}{ Number of layers } \\
\hline & \multicolumn{2}{|l|}{10} & \multicolumn{2}{|l|}{20} & \multicolumn{2}{|l|}{40} & \multicolumn{2}{|l|}{100} & \multicolumn{2}{|l|}{200} & \multicolumn{2}{|l|}{400} \\
\hline & $t(\mathrm{sg})$ & $E(\%)$ & $t(\mathrm{sg})$ & $E(\%)$ & $t(\mathrm{sg})$ & $E(\%)$ & $t(\mathrm{sg})$ & $E(\%)$ & $t(\mathrm{sg})$ & $E(\%)$ & $t(\mathrm{sg})$ & $E(\%)$ \\
\hline 0.01 & 0.183 & 100 & 0.230 & 100 & 0.338 & 100 & 0.676 & 38.40 & 1.216 & 11.02 & 2.328 & 2.024 \\
\hline 0.10 & 0.230 & 43.09 & 0.308 & 11.23 & 0.488 & 1.893 & 1.021 & 0.984 & 1.853 & 0.331 & 3.550 & 0.113 \\
\hline 0.40 & 0.348 & 1.900 & 0.503 & 1.204 & 0.889 & 0.336 & 2.047 & 0.107 & 3.946 & 0.037 & 7.796 & 0.013 \\
\hline 0.80 & 0.463 & 2.191 & 0.784 & 1.005 & 1.475 & 0.295 & 3.480 & 0.075 & 6.750 & 0.027 & 13.405 & 0.010 \\
\hline 1.40 & 0.578 & 4.234 & 1.019 & 1.659 & 1.898 & 0.614 & 4.551 & 0.160 & 8.953 & 0.057 & 17.761 & 0.020 \\
\hline 2.00 & 0.591 & 4.234 & 1.019 & 1.659 & 1.893 & 0.614 & 4.567 & 0.160 & 8.967 & 0.057 & 17.736 & 0.020 \\
\hline
\end{tabular}


Table 6

Rectangular section: Results from the analytical integration method

\begin{tabular}{|c|c|c|c|c|}
\hline \multirow[t]{3}{*}{$\alpha$} & \multicolumn{4}{|c|}{ Time $(t(\mathrm{sg}))$} \\
\hline & \multicolumn{2}{|l|}{$\beta=15^{\circ}$} & \multicolumn{2}{|l|}{$\beta=45^{\circ}$} \\
\hline & $\begin{array}{l}\text { Unique } \\
\text { equations }\end{array}$ & $\begin{array}{l}\text { Separated } \\
\text { components }\end{array}$ & $\begin{array}{l}\text { Unique } \\
\text { equations }\end{array}$ & $\begin{array}{l}\text { Separated } \\
\text { components }\end{array}$ \\
\hline 0.01 & 14.32 & 1.723 & 15.12 & 1.723 \\
\hline 0.10 & 14.22 & 1.703 & 14.92 & 1.753 \\
\hline 0.40 & 15.12 & 3.666 & 14.72 & 1.673 \\
\hline 0.80 & 14.72 & 3.695 & 15.02 & 5.628 \\
\hline 1.40 & 14.92 & 9.083 & 14.32 & 9.133 \\
\hline 2.00 & 14.53 & 9.153 & 15.12 & 9.103 \\
\hline
\end{tabular}

Table 7

Circular section: Results from the analytical integration method

\begin{tabular}{ll}
\hline$\alpha$ & Time $(t(\mathrm{sg}))$ \\
\hline 0.01 & 1.841 \\
0.10 & 1.833 \\
0.40 & 1.744 \\
0.80 & 1.747 \\
1.40 & 1.760 \\
2.00 & 1.785 \\
\hline
\end{tabular}

performed with a unique function, the execution time is seven times slower than for the numerical integral with three Gauss points (Table 2). However, if the analytical integral is performed by separated components, the computing time decreases considerably, the numerical integral being between 0.8 and 2.7 times faster.

Table 7 presents the time for the analytical integral and the circular section. It can be shown that the time of the numerical integral is slightly lower than the analytical one with 15 Gauss points per layer.

In order to compare the three integration methods applied to the circular section, Fig. 9 displays the error for the axial load (Eq. (18)) on the $y$-axis, and the computation time on the $x$-axis for the average value of the five series. The results from the numerical and analytical methods are included in this graph for the following relative depths of the neutral axis $(\alpha): 0.01 ; 0.10 ; 0.20$ and 0.80 . The average computing integration time corresponding to the cases performed with the analytical method is also represented with a continuous vertical line (1.76 sg).

As is shown in Fig. 9, if the integration is performed using the layer method, the time increases as the relative depth increases because there is a higher number of layers in the compressed area. Moreover, if the proposed numerical method is compared with the layer method, the former is found to be more efficient.

Finally, it can be inferred that for a level of errors that could be considered to be admissible in a non-linear program (between $0.01 \%$ and $0.001 \%$ ) the computing time of the numerical method is similar to that of the analytical method.

\section{Conclusions}

This paper presents a comparative study of different analytical and numerical methods in order to determine which is the most efficient algorithm to compute the concrete interaction surface for rectangular and circular sections.

Two new methods, one analytical and the other numerical, have been proposed for the circular sections. The analytical method proposed in this paper obtains the stress integral by using Heaviside functions to define the failure of the section. Moreover, the numerical method proposed here decomposes the integration area into thick layers parallel to the neutral axis. The definition of such layers depends on the concrete constitutive equation used. The internal forces $\left(N_{\mathrm{c}}, M_{\mathrm{c} y}, M_{\mathrm{c} z}\right)$ are computed as a sum of the numerical integrals using a Gauss-Legendre quadrature for each layer the compressed concrete zone is decomposed into.

In general, it was observed that the level of accuracy of the fiber method or layers depends on the mesh density and how it adapts to the compressed concrete zone.

For the rectangular section, the most efficient integration method is the "Modified Thick Layer Integration"

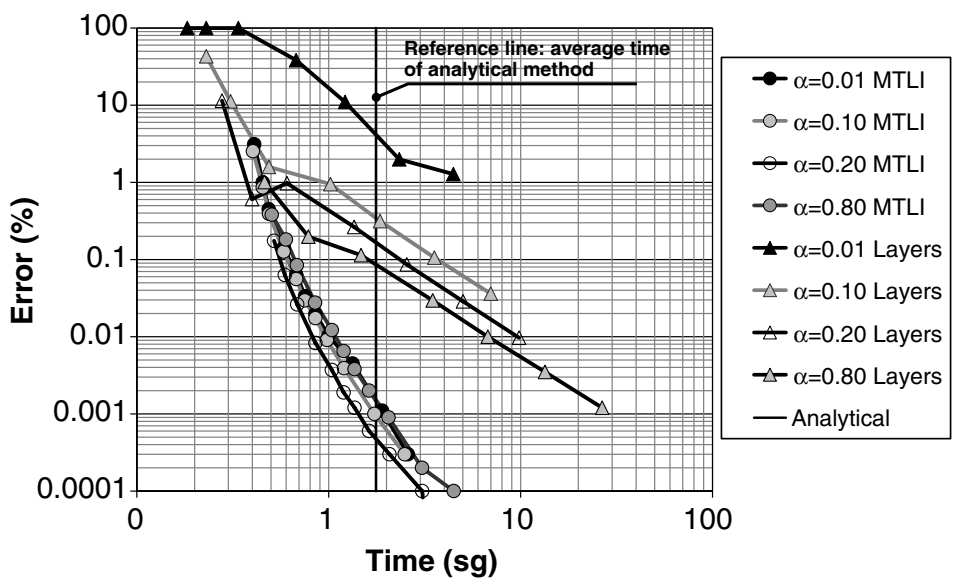

Fig. 9. Circular section: comparative study of the efficiency. 
(MTLI) method with regard to the analytical method and the fiber method. If the parabola-rectangle diagram is selected, three Gauss points are suggested for each side of the perimeter for each layer the compressed zones are broken down into.

For the circular section, both the analytical method and the numerical method are very efficient in comparison with the layer integration method. If an error below $0.04 \%$ is accepted, the numerical method with 15 Gauss points for each layer is recommended.

Consequently, the integration methods based on the Gauss-Legendre quadrature that decompose the compressed are into thick layers parallel to the neutral axis become very effective when it comes to computing the interaction diagram of the concrete section. The optimisation of this procedure gives rise to a notable decrease in the computation times in the design programs that require an iterative process to obtain the reinforcement bars of the section.

\section{Acknowledgements}

This paper was supported by the Spanish "Ministerio de Fomento" under project BOE 13/12/2002 by FCT, Portuguese Minister of Science and Technology, Programa Operacional do Quadro Comunitário de Apoio (POCTI), and by FEDER, by grant POCTI /ECM/12126/2001.

\section{References}

[1] Barros MHFM, Barros A, Ferreira C. Closed form solution of optimal design of rectangular reinforced concrete sections. Eng Comput 2004;21(7):761-76.
[2] Bonet JL, Romero ML, Miguel PF, Fernandez MA. A fast stress integration algorithm for reinforced concrete sections with axial loads and biaxial bending. Comput Struct 2004;82(2-3):213-25.

[3] Romero ML, Miguel PF, Cano JJ. A parallel procedure for nonlinear analysis of reinforced concrete three dimensional frames. Comput Strut 2002;80(16-17):1337-50.

[4] Virdi KS, Dowling PJ. Ultimate strength of composite columns in biaxial bending. Proc Inst Civ En (London) 1973;55(Part 2):251-72.

[5] Mari AR. Nonlinear geometric, material and time dependent analysis of three dimensional reinforced and prestressed concrete frames. Report No. USB/SESM-84/12, Department of Civil Engineering, University of California, Berkley, California, USA, June 1984.

[6] Miguel PF, Bonet JL, Fernández MA. Integración de tensiones en secciones de hormigón sometidas a flexocompresión esviada. Revista Internacional de Métodos Numéricos para el cálculo y diseño en ingeniería 2000;16(2):209-25 [in Spanish].

[7] Basu AK, Suryanarayana P. Reinforced concrete columns under biaxial bending. Int J Struct 1982;2(3):99-114.

[8] Brondum T. Ultimate limit states of cracked arbitrary cross sections under axial loads and biaxial bending. ACI Concr Int 1982;4(11):51-5.

[9] Brondum T. Ultimate flexural capacity of cracked polygonal concrete sections with circular holes under biaxial bending. ACI Concr Int 1987;84(3):212-5.

[10] Yen JR. Quasi-Newton method for reinforced concrete column analysis and design. J Struct Eng, ASCE 1991;117(3):657-66.

[11] JA Rodríguez-Gutierrez. Dario Aristizabal-Ochoa J. Biaxial Interaction Diagrams for Short RC Columns of Any Cross Section. J Struct Eng, ASCE 1999;125(6):672-83. June.

[12] Barros MHFM, Ferreira CC, Barros AFM. Integração do diagrama de tensões de compressão do betão em flexão desviada usando a equação do MC90. Construlink, www.construlink.com, 2003; Vol.1 N. 3, pp 41-49.

[13] Fafitis A. Interaction surfaces of reinforced-concrete sections in biaxial bending. J Struct Eng, ASCE 2001;127(7):840-6.

[14] Comité Euro-internacional du beton: CEB-FIB Model Code 1990 C.E.B. Bulletin No. 203-204 and 205; 1991.

[15] European Committee for Standardization: Eurocode 2: Design of concrete structures- Part 1: general rules and rules for buildings. EN 1992-1-1 December 2004. 